Valoración del impacto económico y social del Carnaval de Negros y Blancos de Pasto, Colombia

Mario Hidalgo 
Lecturas de Economía, 90 (enero-junio 2019), pp. 195-225

\title{
Mario Hidalgo
}

\section{Valoración del impacto económico y social del Carnaval de Negros y Blancos de Pasto, Colombia}

Resumen: El Carnaval de Negros y Blancos que se realiza en la ciudad de Pasto, Colombia, fue declarado por la Unesco como Patrimonio Cultural Inmaterial de la Humanidad en 2009. El objetivo de este articulo es evaluar el impacto económico y social de este carnaval utilizando datos de la versión llevada a cabo en 2017. Con este fin, se utilizan algunos de los instrumentos analíticos de la economía de la cultura, como los métodos de análisis input-output, análisis costo-beneficio, el método de valoración contingente y matrices de valoración cualitativa. El uso de estos instrumentos permite conchir que este carnaval es rentable económica y socialmente, pues expande la capacidad productiva local, genera riqueza material, crea nuevos puestos de trabajo y aumenta la inversión.

Palabras clave: Carnaval de Negros y Blancos de Pasto, análisis de costo-beneficio, economía de la cultura, bienes culturales.

Clasificación JEL: D61, D70, H41, Z10.

Valuing the economic and social impact of the Carnival of Blacks and Whites of Pasto, Colombia

\begin{abstract}
The Carnival of Blacks and Whites held in the city of Pasto, Colombia, was inscribed by UNESCO on the Representative List of the Intangible Cultural Heritage of Humanity in 2009. The objective of this article is to evaluate the economic and social impact of this carnival using data from the 2017 version. To this end, some of the analytical tools of the economics of culture are used, such as the methods of input-output analysis, cost-benefit analysis, the contingent valuation method and matrices of qualitative assessment. Use of these analytical tools allow concluding that this carnival is profitable both economically and socially because it expands the local productive capacity, generates material wealth, creates new jobs and increases investment.
\end{abstract}

Keywords: Carnival of Blacks and W bites of Pasto, cost-benefit analysis, economics of culture, cultural public goods.

JEL Classification: $D 61, D 70, H 41, Z 10$.

\section{Évaluation de l'impact économique et social du Carnaval des Noirs et Blancs à Pasto, Colombie}

Résumé: Le Carnaval des Noir et Blanc de la ville de Pasto (Colombie), a été déclaré Patrimoine Culturel Immatériel de l'Humanité par l'Unesco en 2009. L'objectif de cet article est d'évaluer l'impact économique et social de ce carnaval à l'aide des données recueillies pendant la version de 2017. À cette fin, certains des instruments analytiques de l'économie de la culture sont utilisés, tels que les méthodes d'analyse inputoutput, l'analyse coûts-avantages, la méthode d'évaluation contingente et les matrices de évaluation qualitative. L'utilisation de ces instruments nous permet de conclure que ce carnaval est économiquement et socialement rentable puisqu'il accroît la production locale, crée de la richesse matérielle, crée de nouveaux emplois et accroît les investissements.

Mots-clés: Carnaval des Noirs et Blancs, analyse coûts-avantages, économie de la culture, biens culturels.

Classification JEL: D61, D70, H41, Z10. 


\title{
Valoración del impacto económico y social del Carnaval de Negros y Blancos de Pasto, Colombia
}

\author{
Mario Hidalgo ([- ${ }^{\mathrm{a}}$ \\ -Introducción. -I. Aspectos metodológicos. -II. Caracterización del Carnaval de Negros y \\ Blancos de Pasto. -III. Valoración económica y social del Carnaval. -Conclusiones. \\ -Referencias
}

doi: 10.17533/udea.le.n90a07

Primera versión recibida el 6 de febrero de 2018; versión final aceptada el 10 de octubre de 2018

\section{Introducción}

El Carnaval de Negros y Blancos de Pasto (Colombia), declarado por la Organización de las Naciones Unidas para la Educación, la Ciencia y la Cultura (Unesco) en 2009 como Patrimonio Cultural Inmaterial de la Humanidad, es una festividad eminentemente popular. Representa un espacio de encuentro cultural donde, año tras año, se reúnen propios y visitantes para compartir y jugar en torno al cosmético y al polvo como elementos esenciales del Carnaval. También es un espacio para contemplar y disfrutar de los majestuosos desfiles y eventos culturales que son bienes públicos resultantes del ingenio y de la creatividad de cientos de artesanos y cultores de esta festividad popular.

Aunque el Carnaval ha sido analizado desde diferentes enfoques, poca importancia se le ha prestado a su dimensión económica, en términos de producción de bienes y servicios culturales, riqueza material y generación de puestos de trabajo. En este artículo, novedoso y original, se analiza el impacto económico y social del Carnaval de Negros y Blancos de Pasto en su versión 2017,

a Mario Eduardo Hidalgo Villota: profesor de tiempo completo adscrito al Departamento de Economía e integrante del Grupo de Investigación en Desarrollo Regional (IDER) del Centro de Estudios de Desarrollo Regional (CEDRE), Facultad de Ciencias Económicas y Administrativas de la Universidad de Nariño, Colombia. Dirección postal: Campus Universitario Torobajo, Pasto, Colombia. Dirección electrónica: mariohidalgo@udenar.edu.co.

https://orcid.org/0000-0002-5579-6136 
usando instrumentos de la teoría económica, en particular, de la economía de la cultura.

Aquí se da respuesta a una pregunta recurrente entre las autoridades públicas, Corpocarnaval, los cultores del Carnaval y de la sociedad pastusa: $\dot{e}$ Es el Carnaval de Negros y Blancos de Pasto un buen negocio para el municipio de Pasto? Con el apoyo de instrumentos y técnicas de análisis económico cuantitativo como el método de costo-beneficio, complementado con el análisis input-output, el método de valoración contingente y matrices de análisis cualitativo, se pudo corroborar que el Carnaval es una excelente alternativa de inversión cultural, pues el retorno de la misma, expresado en beneficios económicos, supera con creces los costos y gastos efectuados por Corpocarnaval en las fases de planeación, organización y puesta en escena.

\section{Aspectos metodológicos}

La valoración del impacto económico y social del Carnaval, se hizo a partir del análisis de las bases de datos resultantes de la investigación realizada por el Centro de Estudios de Desarrollo Regional (CEDRE) de la Universidad de Nariño (2017). Dicha investigación se realizó en el marco del proyecto: "Identificación de oportunidades de empleo y emprendimiento en torno al Carnaval de Negros y Blancos de Pasto", propuesta ejecutada en el marco del Observatorio Regional del Mercado de Trabajo de Nariño, la cual contó con el apoyo financiero del Programa de Naciones Unidas para el Desarrollo, la Secretaría de Desarrollo Económico de Pasto, la Dirección de Cultura Departamental de Nariño y la Cámara de Comercio de Pasto.

La recolección de información primaria relevante se hizo a partir de la aplicación de 400 encuestas a espectadores locales del Carnaval residentes en la ciudad de Pasto. Las encuestas se realizaron mediante muestreo aleatorio estratificado durante los meses de noviembre y diciembre de 2016, con una cobertura de 142 barrios de la ciudad de Pasto y a partir de la determinación de submuestras estadísticas por estrato socioeconómico. La determinación de la muestra de espectadores culturales se hizo con base en la población total del municipio de Pasto y su distribución por estratos socioeconómicos. 
Además, se realizaron 286 encuestas $^{1}$ a turistas tanto nacionales como extranjeros en el periodo comprendido entre el 28 de diciembre de 2016 (momento en que inicia la etapa de pre-carnaval con la apertura de Arcoíris en el Asfalto) y el 7 de enero de 2017 (momento en que culmina con la celebración del Festival del Cuy y la Cultura Campesina). La identificación y la toma de información a turistas contó con el apoyo del recurso humano al servicio de la Secretaría de Desarrollo Económico de Pasto, tal como se muestra en la Tabla 1.

Tabla 1. Ficha técnica de la investigación

\begin{tabular}{|c|c|c|}
\hline Características & $\begin{array}{l}\text { Encuesta 1: Espectadores } \\
\text { culturales locales }\end{array}$ & $\begin{array}{l}\text { Encuesta 2: Visitantes } \\
\text { nacionales y extranjeros }\end{array}$ \\
\hline Universo & $\begin{array}{l}\text { Residentes en la ciudad de Pasto } \\
\text { mayores de } 18 \text { años de edad }\end{array}$ & $\begin{array}{l}\text { Turistas nacionales y extranjeros } \\
\text { que visitan la ciudad de Pasto en } \\
\text { temporada de carnaval mayores } \\
\text { de } 18 \text { ańos de edad }\end{array}$ \\
\hline Unidad muestral & Persona & Persona \\
\hline Ámbito geográfico & Ciudad de Pasto & $\begin{array}{l}\text { Ciudad de Pasto - Eventos } \\
\text { culturales del carnaval }\end{array}$ \\
\hline $\begin{array}{l}\text { Método de recolección } \\
\text { de información }\end{array}$ & Encuesta personal & Encuesta personal \\
\hline $\begin{array}{l}\text { Procedimiento de } \\
\text { muestreo }\end{array}$ & $\begin{array}{l}\text { Muestreo aleatorio estratificado } \\
\text { por estrato socioeconómico }\end{array}$ & Muestreo aleatorio simple \\
\hline Tamaño de la muestra & 339 & 263 \\
\hline Nivel de confianza & $95 \% ; Z=1,96 ; p=q=50 \%$ & $95 \% ; Z=1,96 ; p=q=50 \%$ \\
\hline $\begin{array}{l}\text { Fecha de trabajo de } \\
\text { campo }\end{array}$ & Noviembre y diciembre de 2016 & $\begin{array}{l}28 \text { de diciembre a } 7 \text { de enero de } \\
2017\end{array}$ \\
\hline
\end{tabular}

Fuente: elaboración propia.

1 Como se desconoce la población de turistas nacionales y extranjeros que visitan la ciudad de Pasto en temporada de carnaval, se calculó la muestra por el método de poblaciones infinitas con un error muestral de $5,8 \%$. 
A pesar de que existentes variados métodos y procedimientos de análisis, debido a la limitada información de carácter cuantitativo, la evaluación del impacto económico y social del Carnaval se hizo mediante la aplicación del análisis costo-beneficio (ACB), complementado con el análisis input-output (AIO). Además, se tomó como insumo el sistema de cuentas económicas departamentales del periodo 2000-2016 del Departamento Administrativo Nacional de Estadística (DANE) y el sistema de cuentas económicas del municipio de Pasto, elaborado por Ruano et al. (2012) y publicado por la Universidad de Nariño.

La valoración monetaria del Carnaval se efectuó a través del cálculo de la disposición a pagar (DAP) por parte de los espectadores culturales del Carnaval, y se apoyó en el método de valoración contingente (MVC). Mediante este último método se simula la creación de un mercado hipotético en el que el público juega como en mercado real y puede proporcionar, entonces, su DAP en valor monetario, concepto equivalente al de precio explícito utilizado en mercados reales. En este caso, la encuesta realiza el papel de mercado contingente, donde la oferta viene representada por la persona que entrevista (el encuestador) y la demanda por la persona entrevistada (el encuestado); a continuación, se realiza una subasta hasta que se determine la disposición a pagar por parte de los demandantes, en este caso, los espectadores culturales.

Aunque no existe un registro oficial o privado que dé cuenta del número de visitantes nacionales y extranjeros que visitan la ciudad de Pasto para participar en el Carnaval, se estimó a partir de ciertas preguntas, por ejemplo: “En los últimos cinco años, en su vivienda han hospedado a turistas en época del Carnaval?". En total, se estima que 169.443 personas participan en esta festividad en 2017, de los cuales 150.336 son espectadores locales y 19.077 turistas nacionales y extranjeros.

Como la valoración del impacto económico del Carnaval debe ser medida a través de la estimación del gasto de consumo, en las encuestas se incluyeron preguntas específicas que indagan al encuestado sobre variables como el gasto en transporte, alojamiento, alimentos, bebidas, entre otras. 


\section{Caracterización del Carnaval de Negros y Blancos de Pasto}

\section{A. Una macro festividad popular}

En la ciudad de San Juan de Pasto, al sur de Colombia, entre el 28 de diciembre y el 7 de enero de cada año, se celebra el Carnaval de Negros y Blancos de Pasto, festividad cultural auténtica del pueblo pastuso.

El Carnaval de Negros y Blancos de Pasto es la fiesta de todas y todos en comunión con libertad, juego, arte, creatividad, alegría y amor. Es la necesidad del ritual histórico que tienen los pastusos de resistir al tedio, sublimar el trabajo del artesano y soñar con otro tiempo y espacio que les permite ser desde el sentimiento, orgullo y reconocimiento para un pueblo de artistas, cultores, artesanos y trabajadores del campo. (Ministerio de Cultura \& Alcaldía Municipal de San Juan de Pasto, 2010, p. 3).

La importancia de este Carnaval ha sido reconocida por varias entidades, tanto al interior de Colombia como en el exterior. Así, el Congreso de Colombia, mediante la Ley 706 del 26 de noviembre de 2001, declaró el Carnaval de Negros y Blancos de Pasto como patrimonio cultural de la Nacional. Así mismo, el Ministerio de Cultura, a través de la Resolución No. 1557 del 24 de septiembre de 2007, declaró este carnaval pastuso como bien de interés cultural de la Nación. Finalmente, el 30 de septiembre de 2009, el Carnaval de Negros y Blancos fue reconocido por la Unesco como Patrimonio Cultural Inmaterial de la Humanidad.

En la Convención para la Salvaguardia del Patrimonio Cultural Inmaterial, la Unesco definió el Patrimonio Cultural Inmaterial como "los usos, representaciones, expresiones, conocimientos y técnicas -junto con los instrumentos, objetos y artefactos y espacios culturales que le son inherentes-, que las comunidades, los grupos y algunos casos los individuos reconozcan como parte integrante del patrimonio cultural" (Unesco, 2003). Este patrimonio, que se transmite de generación en generación, es creado constantemente por las comunidades y grupos en función de su entorno, su interacción con la naturaleza y su historia, lo que les infunde un sentimiento de identidad y continuidad y contribuye a promover el respeto por la diversidad cultural y la creatividad humana. 
El Carnaval de Negros y Blancos de Pasto es, sin duda alguna, un dinamizador del turismo cultural, tanto en el municipio de Pasto como en el resto del departamento de Nariño. En su proceso de internacionalización, el Carnaval acoge a miles de turistas procedentes de muchos países del mundo; así mismo, en repetidas ocasiones, varios cultores del Carnaval han mostrado la riqueza cultural de esta celebración realizando fiestas similares en otros países.

\section{B. Un bien económico y cultural}

Para Ávila y Díaz (2001), el concepto de "cultura" es un concepto de importancia creciente que también contribuye al desarrollo económico y a la consolidación de una auténtica sociedad del ocio. Por su parte, Herrero (2001), resalta que la economía de la cultura y su interés por relacionar los aspectos culturales y económicos es reciente. Adam Smith (1776), considerado como el padre de la economía clásica, pensaba que las profesiones dedicadas al arte, la cultura y el esparcimiento no contribuían a la riqueza de las naciones, sino que, por el contrario, pertenecían al ámbito del trabajo improductivo. En su obra cumbre, La riqueza de las naciones, así lo manifestó:

[...] En la misma clase [la de los trabajadores no productivos] deben colocarse otras muchas profesiones, tanto de las más importantes y graves, como de las más inútiles y frívolas: los jurisconsultos, los médicos, los hombres literatos de todas especies, clase muy importante y muy honrada; y los bufones, jugueteros, músicos, operistas, bailarines, figurantes, etcétera, que son de una ínfima jerarquía. El trabajo del mínimo de ellos [...] nada produce que sea capaz por su valor real y permanente de comprar o adquirir igual cantidad de otro trabajo; porque perece en el momento mismo de su producción, como la declamación de un actor, la arenga de un orador, o el tono de un cantarín (Smith, 1794 Libro II, Cap. III, p. 99).

Aguado, Palma y Pulido (2017) afirman que Baumol y Bowen, con su investigación Performing arts: The economic dilemma (1966), fueron los primeros economistas en aproximarse al estudio del mundo artístico y cultural, en su 
caso particular, a las artes escénicas como bien cultural. Estos autores concluyeron que el costo de producir dicho bien crecía por encima de los otros sectores de la economía y que, además, solo una pequeña parte de la población lo demandaba, por lo tanto, la demanda de bienes de este tipo debería ser financiada a través de recursos públicos. Estos autores afirman que los bienes culturales eran catalogados como bienes raros, excepcionales o poco importantes:

Este tipo de bienes no encajaban en el esquema analítico desarrollado por los economistas en los siglos XVIII y XIX. En efecto, algunas características indican que los bienes culturales no son reproducibles sino de carácter prototípico (p. ej., una pintura) y el fundamento de su valor está en el mensaje simbólico que emiten y que debe ser descifrado por quien los consume (2017, p. 201).

Si bien, en la antigüedad la cultura era considerada como una actividad económica poco valiosa, en términos de crecimiento económico para las naciones, y la relación entre cultura y economía era difusa, hoy en día, las actividades relacionadas con la cultura o sus explotaciones, como empleos del ocio, constituyen un sector económico de enorme dinamismo y el interés por la cultura ha dado un salto cualitativo, de forma que constituye uno de los fenómenos más significativos de la denominada "civilización del ocio"2.

En los últimos años se ha producido un uso creciente del factor cultural como determinante de las políticas de desarrollo local y regional, así como de las estrategias de regeneración urbana (Bianchini \& Parkinson, 1994; Lim, 1993; Scott, 2000). "En efecto, parece que las actividades culturales se consideran como fuentes de riqueza y empleo, eminentemente por la atracción de flujos de gastos y rentas ligados al turismo cultural, pero también por la

2 El consumo cultural representa uno de los múltiples empleos del ocio dentro de una ética absolutamente hedonista de la sociedad contemporánea, que ha cambiado los valores de laboriosidad y producción, típicos de la sociedad industrial del siglo XIX, por los del disfrute del ocio y el tiempo libre. Además, el individuo moderno trata de sujetarse a los elementos de identidad cercanos en el tiempo y en el espacio, frente al desarraigo que impone el fenómeno de la globalización; y esta es la razón del interés inusitado por la historia, el patrimonio cultural y el auge de lo local y lo regional (Herrero, 2001, p. 152). 
transformación y el desarrollo del tejido productivo que, en conjunto, procuran nuevas ventajas comparativas para las ciudades y las regiones" (Devesa et al., 2012, p. 96).

Devesa et al (2009) resaltan que los festivales culturales son fenómenos cada vez más dinámicos en el panorama cultural actual, esto lo demuestra el crecimiento en el número de festivales en muchas ciudades de España y en el resto de Europa; su crecimiento se explica por factores de demanda y oferta, así como por el uso de la cultura como estrategia de desarrollo local y regional. La demanda de bienes culturales es cada vez mayor, según Aguado y Palma (2015) la participación cultural está determinada por la adicción racional y el aprendizaje a través del consumo, como por un conjunto de factores institucionales y tecnológicos que condicionan la valoración social y económica de dicha participación. Palma et al., 2014 resaltan que las fiestas generan beneficios externos tales como el valor estético o espiritual y el valor social, lo que reafirma el sentido de identidad asociado con la ciudad y su vida cultural, su valor histórico, simbólico y de autenticidad.

El Carnaval como patrimonio cultural inmaterial representa una creación cultural con carácter acumulado, con una perspectiva histórica y con un sentido de herencia. Es un evento único que merece ser salvaguardado, pues, como cualquier otra actividad del ocio, vigoriza el consumo cultural. Además, el Carnaval tiene una trascendencia económica, en cuanto que las decisiones sobre el disfrute del tiempo libre responden a preferencias individuales y su uso constituye un bien susceptible de explotación.

\section{Caracterización de los espectadores culturales del Carnaval: residentes y visitantes}

\section{Espectadores culturales residentes en la ciudad de Pasto}

La inmersión de los hogares pastusos en el Carnaval es representativa, en promedio, 3,82 personas por hogar participan en las diferentes manifestaciones del Carnaval. Entre los visitantes del Carnaval, muchos corresponden a familiares y amigos de personas residentes en la ciudad de Pasto, los cuales se alojan en dichos hogares en el periodo de fiestas decembrinas y carnaval. En promedio, en los últimos 5 años, el 16,3\% de los encuestados ha alojado 
a turistas en época del Carnaval; de estos anfitriones, el 85,4\% ha alojado a 0,67 personas en promedio.

El 88,5\% de las personas permanecen en la ciudad de Pasto en la época de Carnaval y el $82,8 \%$ ha participado, al menos, en alguno de los eventos culturales característicos del Carnaval. Es sorprendente observar que el 48,3\% de la población participa en forma permanente año tras año, como espectadores culturales fieles del Carnaval.

Las razones para asistir al Carnaval son múltiples: el 51,1\% de la población lo hace por diversión y esparcimiento, el 20,5\% se complace observando la creatividad de los artesanos y los espectáculos, y el 15,1\% se deleita de la cultura en términos generales. Sin embargo, una minoría del pueblo pastuso prefiere no participar en el Carnaval. El 18,8\% de la población pastusa no se siente atraída por la festividad, el 15,9\% se molesta por el ruido, el polvo y otros aspectos que éste genera; el 13,0\% considera que no hay innovación entre uno año y otro, y el 11,6\% no participan por enfermedad, tal como se muestra en la Tabla 2.

Tabla 2. Participación de los espectadores locales en los eventos del Carnaval, 2017

\begin{tabular}{|c|c|c|c|}
\hline & Evento & Respuestas múltiples & Porcentaje \\
\hline \multirow{2}{*}{ 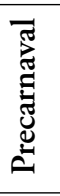 } & Arco iris en el asfalto & 63 & $5,5 \%$ \\
\hline & Desfile de años viejos & 119 & $10,4 \%$ \\
\hline \multirow{7}{*}{ 胥 } & Carnavalito & 159 & $13,9 \%$ \\
\hline & Colectivos coreográficos & 188 & $16,4 \%$ \\
\hline & Familia Castañeda & 169 & $14,7 \%$ \\
\hline & Día de Negros & 96 & $8,4 \%$ \\
\hline & Desfile de Blancos (Desfile Magno) & 284 & $24,8 \%$ \\
\hline & Festival del Cuy & 68 & $5,9 \%$ \\
\hline & Total & 1.146 & $100,0 \%$ \\
\hline
\end{tabular}

Fuente: elaboración propia. 


\section{Espectadores culturales visitantes o turistas}

Aunque se desconoce la cantidad de visitantes o turistas del Carnaval, se logró establecer que el 53,1\% de ellos son hombres y el 46,9\% mujeres. Respecto a la procedencia, el $80,8 \%$ son colombianos que vienen en otros departamentos de Colombia y el 19,2\% son extranjeros que proceden tanto de los Estados Unidos como de países europeos. De otra parte, el 51,7\% son jóvenes en edades comprendidas entre los 23 y los 38 años. Los solteros tienen una participación en el Carnaval de 51,0\%, mientras que los profesionales universitarios, muchos de ellos con estudios de posgrado, representan el $72,4 \%$. El $66,8 \%$ de los espectadores visitantes trabajan y el $42,2 \%$ perciben ingresos mensuales entre uno y tres salarios mínimos colombianos de 2017. El 42,3\% de los turistas afirma haber visitado otros carnavales en Colombia y en el mundo, lo que indica que son personas que sienten atracción por las tradiciones culturales de los pueblos.

El 43,7\% de los turistas justifican su viaje como una oportunidad para presenciar los diferentes eventos y el 33,6\% para visitar familiares y amigos. De otra parte, los turistas no viajan solos, en promedio, lo hacen en pareja, el $53,7 \%$ lo hacen en grupos de hasta 3 personas y el 40,8 \% en grupos de entre 4 y 5 personas. De la población que viaja acompañada, únicamente, el 32,8 \% de los turistas viaja con menores de 12 años de edad (entre 1 y 2 menores). El 52,1\% de los visitantes conocieron del Carnaval a través de contactos con amigos y familiares, el $24,5 \%$ por medio de la televisión y 14,3\% a través de internet. El 89,2\% de los turistas organizó su viaje por sí mismo y únicamente el 2,1\% lo hizo apoyado por agencias de viaje.

En promedio, cada turista permanece en la ciudad de Pasto 3,41 días. El $36,7 \%$ tiene una estadía de hasta 2 días. Durante dichos días, el 62,2\% se hospeda en casas de familiares o amigos, mientras que el 30,8\% lo hace en hoteles y residencias. Entre los diferentes eventos culturales que componen el Carnaval, el 18,5\% de los turistas prefiere el Desfile Magno del 6 de enero por la imponencia de las carrozas, el 14,8\% gustan de la Familia Castañeda, el 14,7 \% del Día de Negros y el 13,7\% de los Colectivos Coreográficos programados para el 3 de enero. La etapa de carnaval ocupa un 89,7 \% de la programación del Carnaval, en comparación con el 10,3 \% que ocupa la etapa 
de pre-carnaval, donde sobresalen eventos como Arco iris en el Asfalto y el tradicional desfile de años viejos (véase Tabla 3).

Tabla 3. Participación de los turistas nacionales y extranjeros en los eventos del Carnaval, 2017

\begin{tabular}{|c|c|c|c|}
\hline & Evento & Respuestas múltiples & Porcentaje \\
\hline \multirow{2}{*}{ 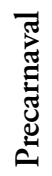 } & Arco iris en el asfalto & 52 & $5,2 \%$ \\
\hline & Desfile de años viejos & 61 & $6,1 \%$ \\
\hline \multirow{7}{*}{ 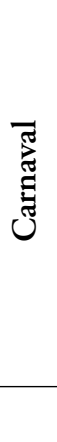 } & Carnavalito & 86 & $8,6 \%$ \\
\hline & Colectivos coreográficos & 151 & $15,2 \%$ \\
\hline & Familia Castañeda & 163 & $16,4 \%$ \\
\hline & Día de Negros & 162 & $16,3 \%$ \\
\hline & Desfile de Blancos (Desfile Magno) & 204 & $20,5 \%$ \\
\hline & Festival del Cuy & 117 & $11,7 \%$ \\
\hline & Total & 996 & $100,0 \%$ \\
\hline
\end{tabular}

Fuente: elaboración propia.

\section{Valoración económica y social del Carnaval}

En este apartado se responde la pregunta “¿cómo actividad cultural popular, es el Carnaval de Negros y Blancos de Pasto un buen negocio para la ciudad de Pasto?". El Carnaval está atado al turismo y, como lo manifiesta Getz (2008), la fuerza motriz económica es claramente el turismo por la dimensión del viaje y los impactos que desencadena. Según Fierro (2014), el turismo es un fenómeno urbano complejo que tiene implicaciones en toda la ciudad de Pasto y en sus estructuras físicas y sociales, las cuales guardan una relación estrecha con el Carnaval. 


\section{A. El Carnaval como generador de empleo}

En los meses de septiembre y diciembre de cada año, el Carnaval es un dinamizador del empleo productivo en ocupaciones como pintores, artesanos, modistas, zapateros, carpinteros, herreros, soldadores, vendedores ambulantes, transportadores, etc. De acuerdo con los cálculos del CEDRE tomando como base la Gran Encuesta Integrada de Hogares (GEIH) en el periodo 2011-2016, el Carnaval generó 8.065 empleos en diversas actividades económicas; esto es, en promedio, unos 1.344 empleos anuales, de los cuales aproximadamente el $20 \%$ se genera en el periodo entre septiembre y diciembre, tal como se muestra en la Tabla 4.

Tabla 4. Empleos directos generados por el Carnaval, 2011-2016

\begin{tabular}{cc}
\hline Año & Número de empleos \\
\hline 2011 & 365 \\
2012 & 1.175 \\
2013 & 1.577 \\
2014 & 1.417 \\
2015 & 2.423 \\
2016 & 1.108 \\
\hline Total & 8.065 \\
\hline Promedio & 1.344 \\
\hline
\end{tabular}

Fuente: CEDRE (2017).

De la población ocupada en el 2016, el 59\% del empleo correspondió a mujeres en actividades ligadas al comercio, tales como la venta de cosméticos, espuma carnavalera, pintura, textiles, alimentos, licores, ponchos, sombreros y gafas, así como en actividades de la industria manufacturera, como la confección de disfraces y vestuario para las murgas y los colectivos coreográficos.

Así mismo, se encontró que, de los ocupados en el mismo año, el 94\% eran trabajadores informales. El empleo formal lo generan empresas del 
sector hotelero, restaurantes y otros servicios. En el año 2016 el Carnaval impactó directamente sobre el empleo en las siguientes ramas de la actividad económica: $8 \%$ en la industria, $50 \%$ en el transporte, $20 \%$ en el comercio, $15 \%$ en hoteles y restaurantes y $7 \%$ en recreación.

Los empleos que generó el Carnaval se discriminan por oficios, así: 50\% como conductores de vehículos de transporte, $20 \%$ como pintores, $15 \%$ para cocineros, camareros, barman y trabajadores similares, el $15 \%$ restante se distribuye en músicos, artistas, empresarios y productores de espectáculos, hilanderos, tejedores, tintoreros, manufactureros y trabajadores similares.

El empleo generado en la actividad del comercio no corresponde, en su totalidad, a la ciudad de Pasto ya que muchos comerciantes de otras regiones del país aprovechan el Carnaval para vender sus productos en esta época. De otro parte, en el caso de la industria, en la región únicamente se estimulan los empleos que tienen relación con la producción textil y artesanal; sin embargo, los cosméticos, sombreros y otros bienes provienen de otras localidades del país (véase Tabla 5).

\section{B. Impacto económico y social del Carnaval}

\section{Enfoque metodológico: análisis costo-beneficio (ACB)}

En la estimación de los costos asociados a la puesta en ejecución del Carnaval se consideraron los costos inherentes al área de la cultura (aportes a la calidad, premiación, eventos del Carnaval, juzgamiento de desfiles, entre otros), área de logística (montaje de tablados para espectáculos, graderías, baños, vallas, transporte, etc.), área de comunicaciones (servicios de comunicación, imagen corporativa, sistema de medios, etc.), área comercial (organización de ruedas de negocios, publicidad, elaboración de productos del Carnaval, etc.) y los costos de servicio de la deuda y gastos de administración. La planeación y ejecución del Carnaval es una actividad de responsabilidad directa de Corpocarnaval. En total, la planeación, administración y montaje del Carnaval 2017 costó \$ 6.159,4 millones, de los cuales el 90,6\% corresponde a costo directo y $9,4 \%$ a gastos de administración. 
Mario Hidalgo: Valoración del impacto económico y social del Carnaval de Negros y Blancos

Tabla 5. Caracterización del empleo generado por el Carnaval

\begin{tabular}{|c|c|c|}
\hline \multicolumn{3}{|l|}{ (a) Población ocupada por sexo } \\
\hline Hombres & 454 & $41,0 \%$ \\
\hline Mujeres & 654 & $59,0 \%$ \\
\hline Total & 1.108 & $100,0 \%$ \\
\hline \multicolumn{3}{|l|}{ (b) Formalidad e informalidad en la población ocupada } \\
\hline Empleos formales & 66 & $6,0 \%$ \\
\hline Empleos informales & 1.042 & $94,0 \%$ \\
\hline Total & 1.108 & $100,0 \%$ \\
\hline \multicolumn{3}{|l|}{ (c) Población ocupada por tipo de oficio } \\
\hline Conductores vehículos de transporte & 554 & $50,0 \%$ \\
\hline Pintores & 222 & $20,0 \%$ \\
\hline Cocineros, camareros, barman y trabajadores asimilados & 166 & $15,0 \%$ \\
\hline Músicos, artistas, empresarios y productores de espectáculo & 66 & $6,0 \%$ \\
\hline Hilanderos, tejedores, tintoreros y trabajadores asimilados & 66 & $6,0 \%$ \\
\hline $\begin{array}{l}\text { Trabajadores manufactureros y trabajadores } \\
\text { asimilados clasificados bajo otros epígrafes }\end{array}$ & 33 & $3,0 \%$ \\
\hline Total & 1.108 & $100,0 \%$ \\
\hline \multicolumn{3}{|l|}{ (d) Población ocupada según parentesco con el Jefe de Hogar } \\
\hline Jefe de hogar & 222 & $20,0 \%$ \\
\hline Cónyuge & 233 & $21,0 \%$ \\
\hline Hijo & 488 & $44,0 \%$ \\
\hline Nieto & 166 & $15,0 \%$ \\
\hline Total & 1.108 & $100,0 \%$ \\
\hline \multicolumn{3}{|l|}{ (e) Población ocupada por rama de actividad } \\
\hline Transporte y comunicaciones & 554 & $50,0 \%$ \\
\hline Comercio & 222 & $20,0 \%$ \\
\hline Hoteles y restaurantes & 166 & $15,0 \%$ \\
\hline Industria & 89 & $8,0 \%$ \\
\hline Recreación & 78 & $7,0 \%$ \\
\hline Total & 1.108 & $100,0 \%$ \\
\hline
\end{tabular}

Fuente: CEDRE con base en la GEIH del DANE.

En el cálculo de los beneficios que genera el Carnaval se consideraron las partidas presupuestales (ingresos recibidos por Corpocarnaval), efectuadas 
por el Ministerio de Cultura, el departamento de Nariño y el municipio de Pasto, que tuvieron un monto $\$ 4.780$ millones, los ingresos obtenidos por la gestión comercial de Corpocarnaval y ventas por concepto de graderías, publicidad y otros por valor de $\$ 1.379,4$ millones (3,5\%); así como los efectos directos, indirectos e inducidos derivados de la matriz input-output $(96,5 \%)$.

En la Tabla 6 se muestra que el Carnaval 2017, requirió una inversión de \$ $6.159,4$ millones y produjo un total de beneficios valorados en $\$ 57.767$ millones, de los cuales 10,7\% corresponden a ingresos y 89,3\% a otros beneficios (efectos directos, indirectos e inducidos). La relación costo-beneficio es de $\$ 9,38$, lo que significa que por cada peso que se invierte en el montaje y la ejecución del Carnaval, este genera $\$ 9,38$ en beneficios (nueve veces su costo de inversión).

\section{Enfoque metodológico: análisis input-output (AIO)}

Aquí se consideran los efectos directos derivados del costo de inversión en el que incurrió Corpocarnaval para garantizar la celebración del Carnaval en su versión 2017, los efectos indirectos derivados del gasto en consumo que los visitantes nacionales y extranjeros realizaron durante su estadía en el Carnaval y los efectos inducidos derivados de la dinámica que generó el impulso de las dos demandas anteriores, mediante las relaciones intersectoriales (compras a otros sectores) y de consumo (nuevos ingresos) en la economía local.

Los efectos directos corresponden a los gastos efectuados por Corpocarnaval en el proceso de planeación y organización del Carnaval, los cuales se estiman en $\$ 6.159,4$ millones, desagregados en ramas económicas tales como: servicios $(62,0 \%)$, industria $(15,9 \%)$, esparcimiento y recreación $(14,5 \%)$, transporte y comunicaciones $(6,7 \%)$, y hoteles y restaurantes $(0,8 \%)$.

De otra parte, los efectos indirectos relacionados con los espectadores locales corresponden al gasto medio en consumo realizado por los residentes en la ciudad de Pasto que participan con regularidad en el Carnaval. Dicho gasto se estima en $\$ 19.298,8$ millones, desagregados en hoteles y restaurantes $(33,9 \%)$, comercio $(26,4 \%)$, industria $(22,8 \%)$, y transporte y comunicaciones $(16,9 \%)$. 
Mario Hidalgo: Valoración del impacto económico y social del Carnaval de Negros y Blancos

Tabla 6. Costos y beneficios del Carnaval, 2017

\begin{tabular}{lrr}
\hline Costos (C) & $\$ 6.159 .438 .072$ & $\mathbf{1 0 0 , 0}$ \\
\hline 1. Área de cultura & $\$ 2.411 .821 .600$ & 39,2 \\
2. Área de logística & $\$ 1.182 .500 .000$ & 19,2 \\
3. Área de comunicación & $\$ 277.369 .250$ & 4,5 \\
4. Área comercial & $\$ 70.105 .320$ & 1,1 \\
5. Administración & $\$ 577.687 .723$ & 9,4 \\
6. Servicio de la deuda & $\$ 1.639 .954 .179$ & 26,6 \\
\hline Beneficios (B) & $\$ 57.766 .779 .257$ & $\mathbf{1 0 0 , 0}$ \\
\hline Efectos directos (organización del carnaval) & $\$ 6.159 .438 .072$ & 10,7 \\
Efectos indirectos (gasto en consumo de residentes) & $\$ 19.298 .783 .322$ & 33,4 \\
Efectos indirectos (gasto en consumo de visitantes) & $\$ 17.316 .794 .795$ & 30,0 \\
Efectos inducidos & $\$ 14.991 .763 .068$ & 26,0 \\
\hline Relación costo/beneficio & $\$ 99,38$ & \\
\hline Fuentes de financiación & Inversión & $\%$ \\
\hline Municipio de Pasto* & $\$ 1.640 .000 .000$ & 42,9 \\
Departamento de Narińo** & $\$ 2.000 .000 .000$ & 32,5 \\
\hline Ministerio de Cultura & $\$ 6.159 .438 .072$ & $\mathbf{1 0 0 , 0}$ \\
\hline Total & & \\
\hline
\end{tabular}

Nota: *Provenientes de recursos del Sistema General de Participaciones (sector cultura), recursos propios y Estampilla Pro-Cultura. ${ }^{* *}$ Provenientes de recursos propios.

Fuente: elaboración propia a partir de la información proporcionada por Corpocarnaval y los métodos de estimación utilizados por el autor.

Aunque no existen registros fiables de las personas que año tras año visitan el Carnaval, a partir del procesamiento de la información recolectada de las encuestas, se estima que en 2017 arribaron a la ciudad de Pasto 19.077 turistas, entre nacionales y extranjeros, de los cuales el $62 \%$ se alojaron en viviendas de familiares o amigos, mientras que el $38 \%$ restante permanecieron en otro tipo de alojamiento. Un visitante hospedado en casas de familiares o amigos permanece, en promedio, 2,46 días durante la etapa del Carnaval (2 a 7 de enero) y realiza un gasto medio en consumo diario de $\$ 309.942$, mientras que un visitante hospedado en otro tipo de alojamiento (hotel, pensión, 
hostal, otros) permanece en promedio, 3,41 días y realiza un gasto medio en consumo diario de $\$ 336.291$.

Los efectos indirectos del Carnaval 2017 se estiman, entonces, en $\$ 17.316,8$ millones, desagregados en ramas económicas tales como: hoteles y restaurantes $(44,4 \%)$, transporte y comunicaciones $(19,4 \%)$, comercio $(18,4 \%)$ y esparcimiento y recreación $(17,8 \%)$.

El aumento de la producción genera nuevos ingresos, los cuales inducen nuevos flujos de gasto y aumento de la producción, y así sucesivamente (efecto inducido), hasta llegar al efecto total sobre el sistema económico local. Los efectos inducidos del Carnaval se estiman en \$14.991,8 millones, desagregados así: la industria (25,8\%), la electricidad, el gas y el agua (15,2\%), el transporte y las comunicaciones $(13,9 \%)$, la rama agropecuaria, de silvicultura, caza, pesca y minería $(13,8 \%)$, el esparcimiento y la recreación $(11,1 \%)$, los servicios $(10,4 \%)$, entre otros.

La Tabla 7 muestra el impacto económico total (efecto directo más efecto indirecto) del Carnaval de Blancos y Negros de Pasto, estimado en $\$ 42.775,0$ millones; además, este genera un efecto inducido de $\$ 14.991,8$ millones, de los cuales el $63 \%$ afecta de manera positiva a la economía del municipio de Pasto y el $37 \%$ a la economía del departamento de Nariño.

Tabla 7. Impacto económico estimado del Carnaval

\begin{tabular}{lrr}
\hline Impacto económico & Monto $(\$)$ & $\%$ \\
\hline Efecto local total & $\mathbf{4 2 . 7 7 5 . 0 1 6 . 1 8 9}$ & 74 \\
Efecto directo & 6.159 .438 .072 & 10,7 \\
Efecto indirecto & 36.615 .578 .117 & 63,4 \\
Efecto inducido total & $\mathbf{1 4 . 9 9 1 . 7 6 3 . 0 6 8}$ & 26 \\
Efecto inducido interior ${ }^{* *}$ & 9.444 .810 .733 & 16,3 \\
Efecto inducido exterior** & 5.546 .952 .335 & 9,6 \\
\hline Impacto económico total & 57.766 .779 .257 & 100 \\
\hline
\end{tabular}

Nota: *Impacto económico sobre la economía del municipio de Pasto. ${ }^{* *}$ Impacto económico sobre la economía del departamento de Narińo.

Fuente: elaboración propia. 
Mario Hidalgo: Valoración del impacto económico y social del Carnaval de Negros y Blancos

Para el cálculo del impacto económico del Carnaval, tanto en el municipio de Pasto como en el departamento de Nariño, se tomaron como referencia los datos del PIB de Pasto (59,2\% del PIB departamental, calculado, en 2016, en $\$ 8.260$ miles de millones de pesos. Con la combinación de las cifras del PIB municipal y departamental para 2016 a precios corrientes más los datos de la Tabla 7, se estimó el tamaño relativo del impacto económico como porcentaje del PIB a nivel municipal y departamental, y se encontró que el Carnaval contribuye con el $0,70 \%$ del PIB del municipio de Pasto y con el $0,41 \%$ del PIB del departamento de Nariño, tal como se muestra en la Tabla 8.

Tabla 8. Tamaño relativo del impacto económico del Carnaval (\% del PIB)

\begin{tabular}{ll}
\hline Impacto económico & $\%$ \\
\hline Aporte al PIB del municipio de Pasto & 0,70 \\
Aporte al PIB del departamento de Nariño & 0,41 \\
\hline
\end{tabular}

Fuente: elaboración propia a partir de las cuentas departamentales elaboradas por el DANE y las Cuentas Municipales de Pasto elaboradas por la Universidad de Nariño y el CEDRE para el 2016 a precios corrientes.

Otra perspectiva de análisis del impacto económico es el uso de multiplicadores medios estimados. El Carnaval tiene un efecto multiplicador total de 0,35 , lo cual significa que por cada peso gastado en su celebración, este produce $\$ 0,35$ adicional, de los cuales $\$ 0,22$ se generan en la economía local (Pasto) y $\$ 0,13$ en la economía del departamento de Nariño ${ }^{3}$, tal como se muestra en la Tabla 9.

En resumen, el efecto local total (directo más indirecto) del Carnaval, estimado en $\$ 42.775,0$ millones, genera un efecto inducido de $\$ 14.991,8 \mathrm{mi}$ llones, de los cuales, el $63 \%$ afecta positivamente a la economía del municipio de Pasto (véase Tabla 10).

3 Según el DANE el PIB del departamento de Nariño en 2016 provisional a precios corrientes se estima en \$ 13.948 miles de millones (1,6\% del PIB nacional). La proyección del sistema de cuentas económicas municipales de Pasto elaborada por el CEDRE de la Universidad de Nariño arroja. para el mismo año, una cifra de $\$ 8.260$ miles de millones. El PIB del municipio de Pasto representa el 59,2\% del PIB departamental y el 0,007 \% del PIB nacional. Para 2016 el PIB de Colombia corresponde a 855.429 miles de millones de pesos corrientes. 
Tabla 9. Multiplicadores medios estimados

\begin{tabular}{lc}
\hline Impacto económico & Multiplicador \\
\hline En comparación con el PIB del municipio de Pasto & 0,22 \\
En comparación con el PIB del departamento de Nariño & 0,13 \\
\hline Total & $\mathbf{0 , 3 5}$ \\
\hline
\end{tabular}

Fuente: elaboración propia.

\section{Enfoque metodológico: método de valoración contingente (MVC)}

Bedate, Herrera y Sanz (2010) consideran que la utilización del método de valoración contingente es bastante frecuente en el campo de los bienes públicos, pero para el caso de los bienes culturales es relativamente reciente. Por su parte, Riera (1994, p. 5) define el MVC como una de las técnicas -a menudo la única - que tenemos para estimar el valor de bienes (productos o servicios) para los que no existe mercado. Es extraordinariamente simple en su comprensión intuitiva: se trata de simular un mercado mediante una encuesta a los consumidores potenciales. Se les pregunta por la máxima cantidad de dinero que pagarían por el bien si tuvieran que compararlo, como hacen con los demás bienes. De ahí se deduce el valor que para el consumidor medio tiene el bien en cuestión.

Para la estimación de la DAP se incluyó en los formularios de la encuesta una pregunta relacionada con la cantidad máxima de dinero que tanto el residente local como el visitante nacional y extranjero estarían dispuestos a pagar para disfrutar de los eventos del Carnaval (desfiles, en especial) por concepto de entrada - en el caso hipotético de que el Carnaval no fuese gratuito-, de acuerdo con lo que han disfrutado (nivel de satisfacción) en su visita y sabiendo que este hecho contribuiría a salvaguardar el Carnaval como patrimonio cultural de la humanidad. Con la inclusión de esta pregunta se pretende simular un mercado hipotético, donde el encuestador actúa como el oferente y el encuestado como el demandante o consumidor (en este caso, un espectador cultural). Como existen personas dispuestas a no pagar $(0=$ respuestas de protesta), la encuesta también indagó los motivos por los que no están dispuestas a pagar. 
Mario Hidalgo: Valoración del impacto económico y social del Carnaval de Negros y Blancos

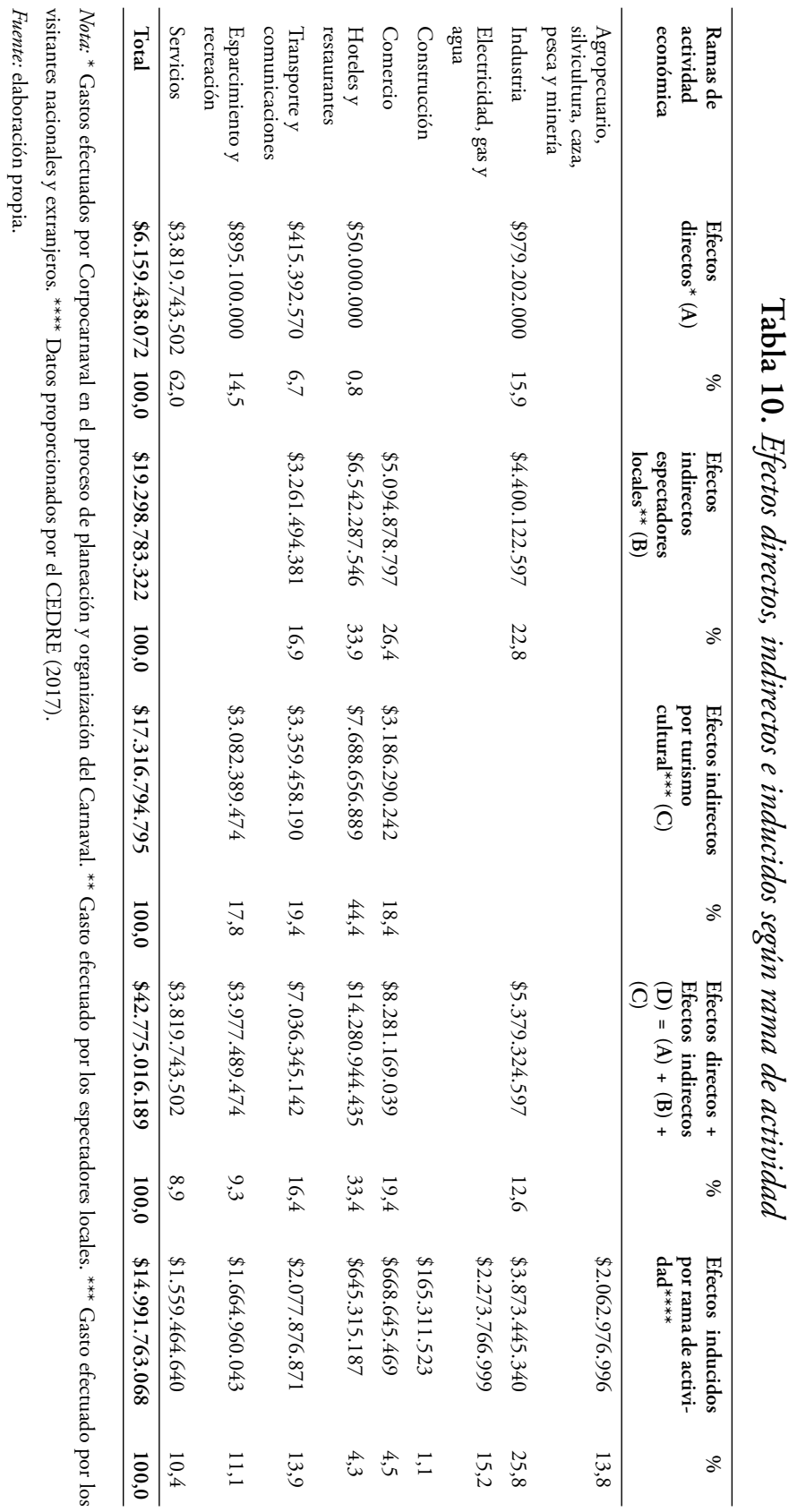


La encuesta arrojó que el $56 \%$ de los espectadores locales están dispuestos a pagar un valor monetario (valor hipotético) por disfrutar de los desfiles durante su estadía en la ciudad de Pasto; en cambio, el $44 \%$ no está dispuesto a pagar ninguna suma de dinero porque consideran que el Carnaval es una festividad popular que debe ser financiada, en su totalidad, con recursos públicos. Un espectador local estaría dispuesto a pagar por desfile y/o evento cultural en el que participa, en promedio, la suma de $\$ 19.549$, ya que disfruta mucho de eventos como el Carnavalito, los colectivos coreográficos, la Familia Castañeda y el Desfile Magno. La mayor DAP la expresan mujeres de estrato medio alto, jóvenes de edades entre 19 y 28 años, profesionales universitarios con postgrado y quienes perciben una renta mensual de entre 4 y $5 \mathrm{SMMLV}^{4}$ de 2017.

El Carnaval de Negros y Blancos es una festividad que despierta lealtad entre los asistentes, lo cual se ve reflejado en el hecho de que el 93,7\% de los visitantes estarían dispuestos a participar nuevamente en él. Así mismo, el $54,2 \%$ manifiesta su disposición a pagar por disfrutar de los desfiles y eventos culturales, mientras que el 45,8\% expresa su negativa por las mismas razones que los espectadores locales. Un visitante nacional y extranjero estaría dispuesto a pagar por desfile y/o evento cultural en el que participa, en promedio, la suma de $\$ 69.012$. La mayor DAP la expresan mujeres, adultos entre los 63 y los 70 años, personas con estudios técnicos y tecnológicos, personas pensionadas y quienes perciben una renta mensual de entre 13 y 14 SMMLV de 2017, tal como se muestra en la Tabla 11.

Tabla 11. Disposición a pagar de locales y turistas

\begin{tabular}{lcccccccc}
\hline $\begin{array}{l}\text { Valoración } \\
\text { económica }\end{array}$ & Media & Mediana & Moda & $\begin{array}{c}\text { Error estándar Desviación Mínimo } \\
\text { de la media }\end{array}$ & Máximo \\
estándar & & \\
\hline DAP Locales & $\$ 19.549$ & $\$ 10.000$ & $\$ 10.000$ & $\$ 1.654$ & $\$ 24.757$ & $\$ 1.000$ & $\$ 200.000$ \\
DAP Visitantes & $\$ 69.012$ & $\$ 30.000$ & $\$ 20.000$ & $\$ 7.169$ & $\$ 89.249$ & $\$ 2.000$ & $\$ 500.000$ \\
\hline
\end{tabular}

Fuente: elaboración propia.

$4 \quad$ El salario mínimo mensual legal vigente (SMMLV) en 2017 fue de \$737.717, equivalente a 250 USD \$ (tomando un tipo de cambio promedio en lo corrido de 2017 de $\$ 2.951,32$ ). 
Desde el enfoque de la economía de la cultura, los eventos culturales del Carnaval (en particular los desfiles) pueden considerarse como bienes públicos, no susceptibles de ser comprados ni vendidos en ningún mercado (sin precio explícito); además, son de carácter colectivo (en cierto modo, bienes no rivales), por lo que no se puede privar a ninguna persona de su uso y disfrute. En este tipo de bienes, al igual que en la mayoría de los disponibles en una economía de mercado, la cantidad demandada varía en forma inversa al precio; es decir, si los eventos culturales del Carnaval tuviesen un precio explícito, los consumidores culturales demandarían una mayor cantidad de aquellos que tienen precios más bajos y los bienes más costos solo estarían al alcance de los consumidores con mayores niveles de renta; por ende, los bienes culturales también son percibidos como bienes sujetos a preferencias individuales y a restricciones presupuestarias.

En la Tabla 12 se muestra que el Carnaval es una inversión cultural rentable. Como se mencionó anteriormente, la planeación y organización de esta festividad requirió una inversión de $\$ 6.159,4$ millones, mientras que los espectadores culturales lo valoran en $\$ 14.318,5$ millones; es decir, los beneficios del Carnaval son el doble de su costo. Por cada peso invertido en la organización y ejecución del Carnaval, este genera $\$ 2,32$ de beneficios.

Tabla 12. Valoración económica del Carnaval

\begin{tabular}{lcccc}
\hline Espectadores culturales & $\begin{array}{c}\text { Número de } \\
\text { personas (A) }\end{array}$ & DAP por evento (B) & $\begin{array}{c}\text { Número de eventos } \\
\text { culturales a demandar (C) }\end{array}$ & $\begin{array}{c}\text { Beneficios } \\
\mathbf{D}=\mathbf{A} \times \mathbf{B} \times \mathbf{C}\end{array}$ \\
\hline Locales & 150.366 & $\$ 20.000$ & 4 & $\$ 12.029 .280 .000$ \\
$\begin{array}{l}\text { Visitantes nacionales y } \\
\text { extranjeros }\end{array}$ & 19.077 & $\$ 30.000$ & 4 & $\$ 2.289 .240 .000$ \\
\hline Total & 169.443 & & $\$ 14.318 .520 .000$ \\
Relación costo/beneficio & & $\$ 2,32$ \\
\hline
\end{tabular}

Fuente: elaboración propia.

Para el cálculo de la DAP se utilizó la mediana, por ser un estadístico más conservador que la media aritmética y los desfiles como bienes culturales. La estimación de los turistas nacionales y extranjeros se hizo con base en preguntas específicas, en la información suministrada por las dependencias e instituciones relacionadas con el turismo y en el cálculo de la capacidad de los 
espacios públicos utilizados como la Senda del Carnaval y otros. También se preguntó a los espectadores culturales acerca de sus percepciones sobre los efectos económicos y sociales del Carnaval, así como sobre el nivel de impacto general que este ejerce sobre la ciudad de Pasto. Se encontró que los residentes locales perciben de forma positiva el Carnaval y consideran que dinamiza en un nivel alto la economía de la ciudad, aumenta el empleo e incrementa el turismo incluso antes del 28 de diciembre (fecha de iniciación del precarnaval); sin embargo, se percibe como aspecto negativo el incremento de los precios de los bienes y servicios en esta temporada (véase Tabla 13).

Tabla 13. Impacto económico percibido por los residentes en la ciudad de Pasto

\begin{tabular}{|c|c|c|c|c|c|c|}
\hline \multirow{2}{*}{ Aspecto } & \multicolumn{6}{|c|}{ Valoración } \\
\hline & Baja & $\%$ & Media & $\%$ & Alta & $\%$ \\
\hline Dinamiza la economía de la ciudad & 21 & 6,2 & 71 & 21,1 & 245 & 72,7 \\
\hline Aumenta el empleo & 57 & 15,9 & 128 & 35,7 & 174 & 48,5 \\
\hline Aumenta el precio de bienes y servicios & 47 & 16,3 & 97 & 33,6 & 145 & 50,2 \\
\hline $\begin{array}{l}\text { Genera escasez de bienes y servicios } \\
\text { (especulación) }\end{array}$ & 51 & 30,2 & 65 & 38,5 & 53 & 31,4 \\
\hline $\begin{array}{l}\text { Genera pérdidas económicas por el cierre de } \\
\text { negocios en la temporada }\end{array}$ & 47 & 25,7 & 72 & 39,3 & 64 & 35,0 \\
\hline $\begin{array}{l}\text { Las ventajas económicas se concentran en } \\
\text { unas pocas personas y empresas }\end{array}$ & 38 & 15,0 & 109 & 43,1 & 106 & 41,9 \\
\hline $\begin{array}{l}\text { Incrementa el turismo antes del } 28 \text { de } \\
\text { diciembre }\end{array}$ & 39 & 15,4 & 50 & 19,7 & 165 & 65,0 \\
\hline Incrementa el turismo después del 6 de enero & 24 & 43,6 & 15 & 27,3 & 16 & 29,1 \\
\hline
\end{tabular}

Fuente: elaboración propia a partir de las respuestas recopiladas en la encuesta.

Los residentes en la ciudad de Pasto también resaltan el impacto social del Carnaval; valoran altamente el reconocimiento y la promoción de la imagen de la ciudad de Pasto, y consideran que representa un orgullo y una satisfacción 
para el pueblo pastuso. Además, consideran que es un elemento que mejora las relaciones personales y el clima social, por lo que se constituye en una oportunidad para promocionar y difundir la cultura pastusa. Como aspecto negativo, la ciudadanía percibe que el Carnaval incrementa la contaminación por el uso desmedido de agua, polvo y espuma carnavalera; aumenta los niveles de inseguridad en la ciudad y genera traumatismos en el tráfico vehicular debido al mayor ingreso de vehículos (véase Tabla 14).

Tabla 14. Impacto social percibido por los residentes en la ciudad de Pasto

\begin{tabular}{|c|c|c|c|c|c|c|}
\hline \multirow{2}{*}{ Aspecto } & \multicolumn{6}{|c|}{ Valoración } \\
\hline & Baja & $\%$ & Media & $\%$ & Alta & $\%$ \\
\hline $\begin{array}{l}\text { Aumenta el reconocimiento y promoción de } \\
\text { la ciudad de Pasto }\end{array}$ & 15 & 4,3 & 63 & 18,1 & 270 & 77,6 \\
\hline $\begin{array}{l}\text { Representa orgullo y satisfacción para los } \\
\text { ciudadanos }\end{array}$ & 34 & 9,2 & 76 & 20,6 & 259 & 70,2 \\
\hline $\begin{array}{l}\text { Mejora las relaciones personales y el clima } \\
\text { social }\end{array}$ & 64 & 22,9 & 112 & 40 & 104 & 37,1 \\
\hline Deteriora el tráfico de la ciudad & 21 & 6,3 & 76 & 22,9 & 235 & 70,8 \\
\hline Genera inseguridad en la ciudad & 32 & 8,8 & 66 & 18,2 & 265 & 73,0 \\
\hline Promociona y difunde la cultura & 24 & 6,7 & 91 & 25,5 & 242 & 67,8 \\
\hline Genera contaminación ambiental & 18 & 4,8 & 56 & 14,9 & 303 & 80,4 \\
\hline
\end{tabular}

Fuente: elaboración propia a partir de las respuestas recopiladas en la encuesta.

Respecto al impacto social del Carnaval, los visitantes nacionales y extranjeros valoran en alto nivel y de manera positiva el acceso a los medios de transporte para llegar a la ciudad y para desplazarse al lugar de los eventos y, por otro lado, valoran en nivel medio las sendas por las cuales se desplazan los desfiles. Finalmente, tienen una percepción baja de la presencia institucional ejercida por Corpocarnaval, la Alcaldía Municipal de Pasto y los organismos de socorro (véase Tabla 15). 
Tabla 15. Impacto social percibido por los visitantes nacionales y extranjeros

\begin{tabular}{lcccccc}
\hline \multirow{2}{*}{ Aspecto } & \multicolumn{7}{c}{ Valoración } \\
\cline { 2 - 7 } & Baja & $\%$ & Media & $\%$ & Alta & $\%$ \\
\hline Organización del evento & 0 & 0,0 & 6 & 1,9 & 3 & 0,9 \\
Seguridad & 4 & 1,3 & 10 & 3,1 & 2 & 0,6 \\
Acceso a transporte & 11 & 3,4 & 22 & 6,9 & 28 & 8,8 \\
Acceso a alimentos & 2 & 0,6 & 11 & 3,4 & 2 & 0,6 \\
Cultura y atención de población local & 5 & 1,6 & 14 & 4,4 & 4 & 1,3 \\
Expresión artística y cultural del evento & 3 & 0,9 & 3 & 0,9 & 2 & 0,6 \\
Espacio para el disfrute de los desfiles (senda & 12 & 3,8 & 23 & 7,2 & 13 & 4,1 \\
del carnaval) & & & & & & \\
Presencia institucional: Policía Nacional & 7 & 2,2 & 5 & 1,6 & 1 & 0,3 \\
Presencia institucional: organismos de & 24 & 7,5 & 5 & 1,6 & 0 & 0,0 \\
socorro (Bomberos voluntarios, Defensa & & & & & & \\
Civil, etc.) & & & & & & \\
Presencia institucional: Corpocarnaval y & 46 & 14,4 & 21 & 6,6 & 9 & 2,8 \\
Alcaldía Municipal de Pasto & & & & & & \\
Presencia institucional: otros actores & 11 & 3,4 & 9 & 2,8 & 1 & 0,3 \\
\hline
\end{tabular}

Fuente: elaboración propia a partir de las respuestas recopiladas en la encuesta.

\section{Conclusiones}

El Carnaval de Negros y Blancos de Pasto fomenta el turismo cultural y, a su vez, incrementa la producción de bienes y servicios relacionados con el ocio, la diversión y la cultura; además, ejerce un efecto multiplicador significativo sobre la economía del municipio de Pasto y de otras localidades con oferta turística conexa, ya que genera nuevos puestos de trabajo antes y durante la realización de esta festividad popular. El estudio demostró que, por cada peso invertido en su organización y ejecución, el Carnaval genera $\$ 9,38$. En este sentido, la relación costo/beneficio medida desde el enfoque del método de valoración contingente es de $\$ 2,32$, esto significa que los espectadores culturales del Carnaval estarían dispuestos a pagar dos veces el costo de la 
Mario Hidalgo: Valoración del impacto económico y social del Carnaval de Negros y Blancos

inversión como valores de opción, de legado y de existencia. La aportación estimada del Carnaval al PIB del municipio de Pasto es de $0,70 \%$ y al PIB del departamento de Nariño es de $0,41 \%$, cifras muy significativas para un bien cultural de este tipo.

El pueblo pastuso considera que esta festividad dinamiza la economía, genera empleo, visibiliza la imagen de la ciudad de Pasto, eleva el nivel de satisfacción y orgullo de los pastusos y mejora las relaciones y el clima social. También se comparte una percepción negativa de que el Carnaval produce efectos inflacionarios de corto plazo, traumatismo en el tráfico vehicular, inseguridad y contaminación por el uso indiscriminado de agua, polvo y espuma carnavalera.

Por otro lado, se encontró que la inversión en las fases de organización y ejecución del Carnaval proviene en una gran proporción del sector público; sin embargo, los agentes económicos privados son los mayores receptores de los beneficios que este produce, paradójicamente, con una baja cuota de aporte.

Como lo evidencia este estudio, el Carnaval es un ícono cultural que se debe preservar y fortalecer; por esto, es indispensable el desarrollo de una política pública que integre actores públicos, privados y cultores del Carnaval y que permita canalizar mayores recursos enfocados hacia la internacionalización de esta festividad popular. Así mismo, Corpocarnaval debe incentivar la participación de nuevos espectadores, preferiblemente jóvenes, entre los cuales se debe fomentar una mayor afición por esta celebración tradicional.

\section{Referencias}

Aguado Quintero, Luis Fernando \& Palma Martos, Luis (2015). "Factores que limitan la participación cultural. Una mirada desde la economía de la cultura", Revista de Ciencias Sociales, Vol. 21, No. 1, pp. 58-71.

Aguado Quintero, Luis Fernando \& Palma Martos, Luis \& Pulido, Noemí (2017). "50 años de economía de la cultura. Explorando sus raíces en la historia del pensamiento económico", Cuadernos de Economía, Vol. 36, No. 70, pp. 197-225. Recuperado de: 
https://revistas.unal.edu.co/index.php/ceconomia/article/view/53813/ 56587 (enero 21 de 2018).

Ávila Álvarez, Antonio María \& Díaz Mier, Miguel Ángel (2001). “Economía de la cultura: ¿una construcción reciente?”, Información Comercial Española, ICE: Revista de economía, No. 792, p. 9-30.

Bedate Centeno, Ana; Herrera Prieto, Luis \& Sanz Lara, José (2007). "Aplicación del método de valoración contingente a bienes culturales. Estimaciones de valor del Museo Patio Herreriano". En Benito Montayo Pedro y Somarriba Arechavala, Noelia (Coords.), Anales de economía aplicada 2007 (Vol. 7, pp. 350-375). Universidad de Valladolid. Recuperado de: https://dialnet.unirioja.es/servlet/articulo?codigo=5169809 (enero 12 de 2018).

Bianchini, Franco \& Parkinson, Michael (Eds.) (1994). Cultural Policy and Urban Regeneration: The West European Experience. Manchester. Manchester: Manchester University Press.

Centro de Estudios de Desarrollo Regional y Empresarial (CEDRE) (2017). Identificación de oportunidades de empleo y emprendimiento en torno al Carnaval de Negros y Blancos de Pasto. Observatorio Regional de Mercado de Trabajo de Nariño. Informe final. Mimeo.

Devesa Fernández, María; Herrero Prieto, Luis y Sanz Lara, José Ángel (2009). "Análisis económico de la demanda de un festival cultural", Estudios de Economía Aplicada, Vol. 27, No. 1, pp. 137-158. Recuperado de: http://giec.blogs.uva.es/files/2012/02/eeadevesaherrerosanz2009.pdf. (Enero 30 de 2018).

Devesa Fernández, María; Baez, Andrea; Figueroa, Víctor y HeRRERO, Luis C. (2012). "Repercusiones económicas y sociales de los festivales culturales: el caso del Festival Internacional de Cine de Valdivia", Revista Latinoamericana de Estudios Urbanos Regionales, Vol. 38, No. 115. pp. 95-115. Recuperado de: https://scielo.conicyt.cl/scielo.php?script=sci_arttext\&pid=S025071612012000300005 (enero 9 de 2018). 
Mario Hidalgo: Valoración del impacto económico y social del Carnaval de Negros y Blancos

Departamento Administrativo Nacional de Estadística (DANE) (s.f.). Dirección de Sintesis y Cuentas Nacionales. Cuentas Departamentales. Disponible en: http://www.dane.gov.co/index.php/estadisticas-portema/cuentas-nacionales/cuentas-nacionales-departamentales (enero 30 de 2018).

Fierro Castro, Cristina (2014). Carnaval de negros y blancos, turismo y transformaciones urbanas en la ciudad de San Juan de Pasto. Trabajo de Grado. Facultad de Ciencias Sociales y Económicas, Universidad del Valle.

Getz, Donald (2008). "Event Tourism: Definition, Evolution, and Research", Tourism Management, Vol. 29, No. 3, pp. 403-428.

Herrero Prieto, Luis César (2001). "Economía del Patrimonio Histórico", Información Comercial Española ICE: Revista de economía, No. 792, pp. 151-168. Recuperado de: http://www.revistasice info/cachepdf/ICE_792_151-168_A11B0C4F6C74891BDA1BF049 B79D894B.pdf. (enero 17 de 2018)

LEY No. 706, Congreso de Colombia, Bogotá, Colombia, 30 de noviembre de 2001.

Lim, Hoc (1993). "Cultural strategies for revitalizing the city: A review and evaluation”, Regional Studies, Vol. 27, No. 6, pp. 589-594.

Ministerio de Cultura de Colombia \& Alcaldía Municipal de San JuAn de PAsto (2010). Plan especial de salvaguardia del Carnaval de Negros y Blancos de Pasto. Documento de sustentación. Recuperado de: http://patrimonio.mincultura.gov.co/SiteAssets/Paginas/PES -Carnaval-de-Blancos-y-Negros/05-Carnaval \%20de \%20negros \%20y \%20 blancos \%20de \%20Pasto \%20- \%20PES.pdf (enero 17 de 2018).

Palma Martos, Luis; Palma Martos, María Luisa; Martín Navarro \& José Luis (2014). "La integración entre cultura y economía. El caso de las Fiestas de Primavera de Sevilla", Estudios de Economía Aplicada, Vol. 32, No. 1, pp. 287-308.

Resolución No. 1557, Ministerio de Cultura, Colombia, 27 de septiembre de 2007. 
Riera, Pere (1994). Manual de valoración contingente. Recuperado de: https:/www.cepal.org/ilpes/noticias/paginas/0/35060/manual_evalua cion_contingente.pdf (Consulta; enero 25 de 2018).

Scott, Allen J. (2000). The cultural economy of cities. London: Sage Publications.

Smith, Adam (1794). Investigación de la naturaleza y causas de la riqueza de las naciones. España: Consejería de Educación y Cultura de la Junta de Castilla y León, Valladolid (obra original publicada en 1776).

Ruano Rosero, Eduardo Vicente; Santacruz, Alejandra; Panesso, Fernando \& Calderón, Felipe Santiago (2012). Cuentas económicas del Municipio de Pasto 2005-2010p. Colombia: Universidad de Narińo.

Unesco (2003). Convención para la salvaguardia del patrimonio cultural inmaterial 2003. Recuperado de: http://portal.unesco.org/es/ev.php-

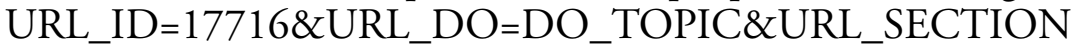
=201.html. (enero 12 de 2018). 